\title{
A Constellation Diagram Design Method with Minimum Average Energy: An Idea from Structural Chemistry
}

\author{
Yan $\mathrm{Li}$ \\ \{yan981i@yeah.net\} \\ College of Communications Engineering, Army Engineering University, \\ Nanjing 210007, China,
}

\begin{abstract}
Based on the theory of crystal structure in chemistry, introducing a method to optimize constellation diagram by means of maximizing space utilization ratio(SUR)when the Euclidean distance between signal points is constant. After studying the average system energy(ASE) and SER of the method and N-QAM system at different points, it is found that the ASE of the method is only $80 \backslash \%$ to $90 \backslash \%$ of that of the latter, and the SER of system based on it is lower when their ASE are the same. These results shows the superiority of the arrangement method in reducing system energy or SER.
\end{abstract}

Keywords: Space Utilization Ratio(SUR), Average System Energy(ASE), SER, Hexagonal Rules

\section{Introduction}

The intuitive representation of signal Constellation Diagram is the modulation state of signal points.[1] The anti-interference ability and energy of communication system are closely related to the arrangement of signal points in its constellation. We often judge the performance of Constellation Diagram by SER and ASE. Generally speaking, the large Euclidean distance between adjacent signal points means that they have less interaction on each other under the influence of noise. So while keeping this distance constant, we can adjust the position of each signal point so that they can be as close as possible to the origin of coordinates, thus having a smaller Average System Energy.

Space utilization is a concept in structural chemistry. It can judge the efficiency of the arrangement of various elementary particles through theoretical analysis. Some arrangements take up more space, while others make good use of space. From this inspiration, we use this concept to explore how to plan the arrangement of signal points in the Constellation Diagrams so that they have the highest energy efficiency, thus reducing the average energy of the system while maintaining the distance between signal points.

QAM has been widely used because of its simple modulation, but the Spatial Utilization Ratio of the signal point arrangement still has a larger space to improve.

The design schemes independently discovered in this paper are partly similar to those in reference [1], but both have the purpose of reducing system energy or improving antijamming capability. Reference[1] introduced a efficient 3-D Constellation Diagram model and examplified how to divide a 3-D Constellation Diagram to several 2-D Constellation 
Diagrams. But did not provide a practical agorithm for caculating 2-D Constellation Diagram. This paper will introduce the principle and method of designing low-energy Constellation Diagram from a new design point of view and concept which is called Spacial Utilization Ratio and give the algorithm of Constellation Diagram design for given points. During the research, we found that it is a good model. Unlike the general literature, it guides our design from the geometric level. At the same time, it has guiding significance for the further interpretation and optimization of the 3-D Constellation Diagram in reference 1. I also hope that more readers can put forward their own ideas, communicate with each other and make progress together. The contributions of this paper are mainly reflected in the following aspects:

- Introduction of SUR to optimize the constellation structure from the geometric level.

- A general algorithm for constellation design based on the principle of minimum energy is presented.

- $\quad$ Exemplify the Hexagonal Arrangement has the highest SUR so that has the lowest ASE.

In this paper, we first introduce the concept of space utilization and explore how to improve it by making the arrangement of signal points more compact without reducing the distance between adjacent points, which analyses the performance of common arrangement modes at the geometric level. Then the hexagonal rule is proposed according to the arrangement method with the maximum space utilization rate, which is the fundamental basis for the constellation design in this paper. Finally, we perform performance analysis. By comparing the method in this paper with the average energy of QAM system under the condition of the same Euclidean distance between adjacent signal points, the superiority of the method in reducing the average energy of QAM system is more quantitatively and intuitively reflected.

\section{Related Work}

People have done a lot of work in the design of Constellation Diagrams in the past. At present, the most common Constellation Diagrams are rectangular QAM Constellation Diagram and cross QAM Constellation Diagram.[2,7] QAM modulation is a very popular modulation method in digital communication. Its efficiency and SER performance are good. At present, QAM modulation is widely used. Its constellation is square. However, the energy of the signal points at each corner of the system is relatively large, which will affect the average energy and maximum energy of the system.[3] So sometimes the points in the corner of the improved QAM constellation are moved closer to the origin of the coordinates to reduce ASE.

In the improvement scheme of Constellation Diagram, the expansion method of Constellation Diagram has also attracted the attention of some researchers. The constraints of this method are to expand constellation points outward without reducing the Euclidean distance between constellation points. It achieves a peak-to-average power ratio about $1 \mathrm{~dB}$ lower than traditional OFDM.[6]

We can see that almost all of above methods are based on QAM or QPSK. The model proposed in this paper makes the design of constellation diagram have a new space for improvement under the viewpoint of these authors. I believe that future authors can make new progress on the basis of the concept of this paper. 
Some people also want to expand the Constellation Diagram to 3-D or even higher dimensions to design. $[4,5,8]$ This is a very creative idea. In our analysis, we can think that this method folds a planar Constellation Diagram so that the signal points are closer to the origin of the coordinates, thus greatly reducing the ASE. For example, in a 3-D Constellation Diagram, the signal points are arranged according to the hexagonal densest accumulation of crystals in chemistry to achieve the maximum space utilization. Of course, the higher the dimension, the higher the degree of folding, and the lower the ASE. However, the geometric properties of high-dimensional constellations intersect with those of planar constellations. Because high dimensional Constellation Diagram can be divided into several lowerdimensional and eventually decomposed into twodimensional Constellation Diagram for analysis.

\section{SPACE UTILIZATION RATIO}

Firstly, we might as well set the minimum distance between adjacent signal points in a constellation as. Then, we can think of the "field" of each signal as a circular region with its center and radius. Then our task is to put as many disjoint circles as possible in a limited area. Next, we analyze two representative methods of ranking.

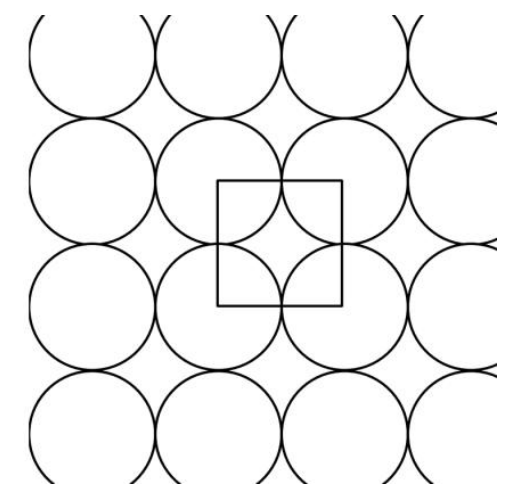

(a)

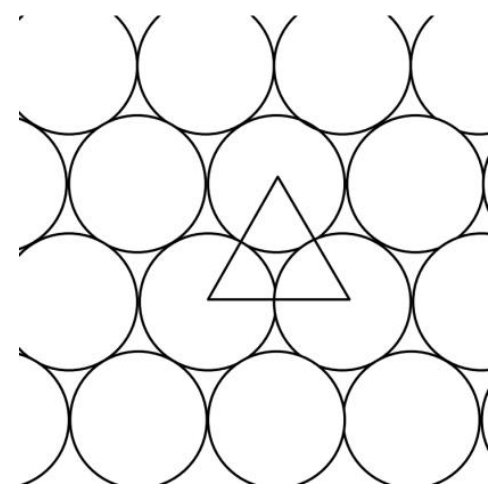

(b)

Fig. 1. Two Arrangement Methods. The subfigure (a) is Square Arrangement; the subfigure (b) is Hexagonal Arrangement

Shown above, in the Square Arrangement in Fig. 1(a), each signal point has four points adjacent to it, which belongs to the square arrangement (N-point QAM is exactly this arrangement). In the Hexagonal Arrangement in Fig. 1(b), each signal point has six nearest adjacent points. The stacking pattern in these two graphs is very similar to that in chemistry. Chemical knowledge tells us that hexagonal arrangement has a higher space utilization rate. Now we will prove it by calculating the space utilization rate of the two arrangements. We can see from the smallest area unit that: 


$$
\eta_{1}=\frac{4 \cdot \frac{1}{4} \pi A^{2}}{(2 A)^{2}}
$$

In the Hexagonal Arrangement:

$$
\eta_{2}=\frac{3 \cdot \frac{1}{6} \pi A^{2}}{A \cdot \sqrt{3} A}
$$

According to formula (1) and (2):

$$
\frac{\eta_{1}}{\eta_{2}}=\frac{\sqrt{3}}{2} \approx 0.8760
$$

At this time, we have come to the conclusion that hexagonal arrangement does have higher space utilization. In addition, this arrangement is the most space-efficient of all the arrangements on the plane. It may be called Planar Closest, hereinafter referred to as PLC. Because a circle's outer regular polygon satisfies the hexagon with the largest number of edges that can be mosaic in the plane, and the area of the outer regular polygon will gradually decrease and approach the area of the circle with the increase of the number of edges.

Similarly, the Spatial Utilization Ratio of permutations in three or higher dimensions can still be achieved using the above methods. And each dimension will have one of the most space-efficient solutions. For example, under 3-D conditions, the Hexagonal Closest Packed, which is shown in Fig.2 ,has the highest Spatial Utilization Ratio rate (about $74 \%)$

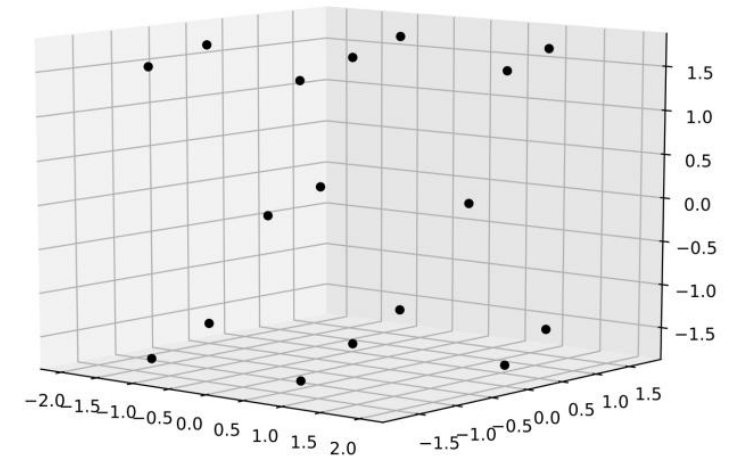

Fig. 2. Hexagonal Closest Packet.

\section{Hexagonal Rules}

Starting from the hexagonal arrangement, we can arrange all the signal points in the intersection points as shown in the Fig 3 . When we select a point on the plane of the 
graph as the center point (not necessarily a black point), the signal points occupy the nearest point to the center first, thus ensuring the lowest energy.

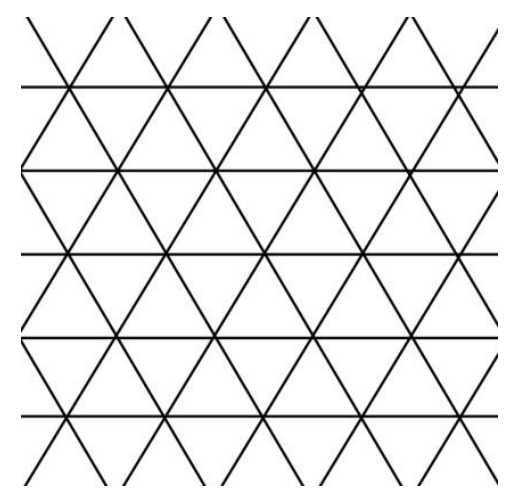

Fig. 3. Equilateral triangular mesh.

Generally speaking, for the choice of the center point, we first briefly analyze: when using the most intensive arrangement, the closer the center of gravity is to the center point,the lower the energy is, which is very similar to the change of inertia of rigid body when choosing different fulcrums. For the convenience of discussion, we set the coordinates of one of the black points to be zero. Four methods of selecting the center point are recommended here. As shown in the Table 1.

Table 1. Four Methods to Select Center Point.

\begin{tabular}{|c|c|c|}
\hline Scheme & Figure & The coordinates of central point \\
\hline I & $\begin{array}{ll}\cdot & \bullet \\
\cdot & \bullet \\
\cdot & \bullet\end{array}$ & $(0,0)$ \\
\hline II & $\begin{array}{ll}\cdot & \bullet \\
\cdot & \cdot \\
\cdot & \bullet\end{array}$ & $(A, 0)$ \\
\hline
\end{tabular}




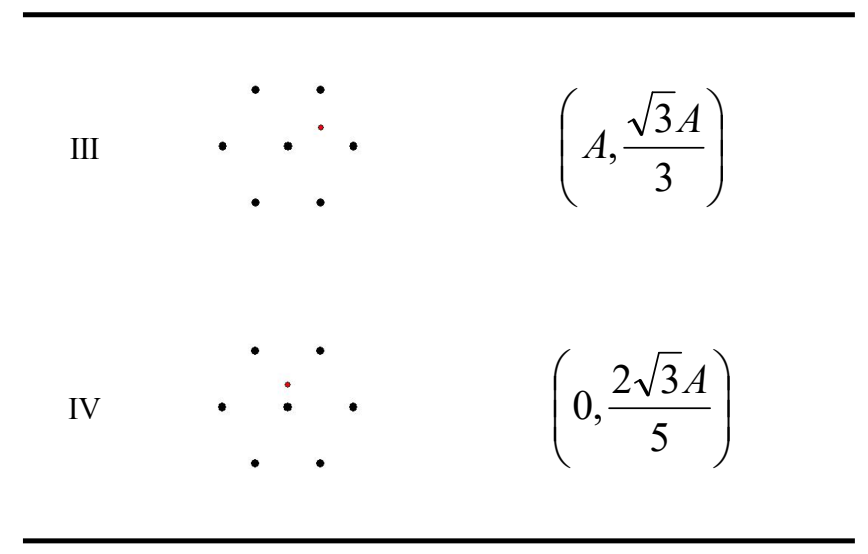

For a given numSER of signal points, we first calculate the average energy E1 (N), E2 $(N), E 3(N), E 4(N)$ of the four permutation methods respectively. Then select the lowest energy arrangement scheme, whose energy is $\min \{\mathrm{E} 1(\mathrm{~N}), \mathrm{E} 2(\mathrm{~N}), \mathrm{E} 3(\mathrm{~N}), \mathrm{E} 4$ (N)\}.Generally speaking, the algorithms for calculating energy and confirming schemes are as follows:

1) Input the number of signal points.

2) Enter enough coordinates of possible signal points on the coordinate plane.

3) For the above four models, the coordinates of the possible signal points are arranged in ascending order according to the distance from the center point, and the first $\mathrm{N}$ coordinate points are selected as the occupied coordinate points.

4) The Average System Energy of the above four methods is calculated by dividing the energy of the first $\mathrm{N}$ points by the number $\mathrm{N}$.

5) The serial number of the lowest energy scheme and its corresponding energy value can be output, and the coordinates of $\mathrm{N}$ signal points can also be output according to the need.

For the above algorithm, the amount of manual calculation will be relatively large. It is suggested that the program be implemented according to the above algorithm.

For example, here we give the Constellation Diagram at 32 and 64 points in Fig. 4. 

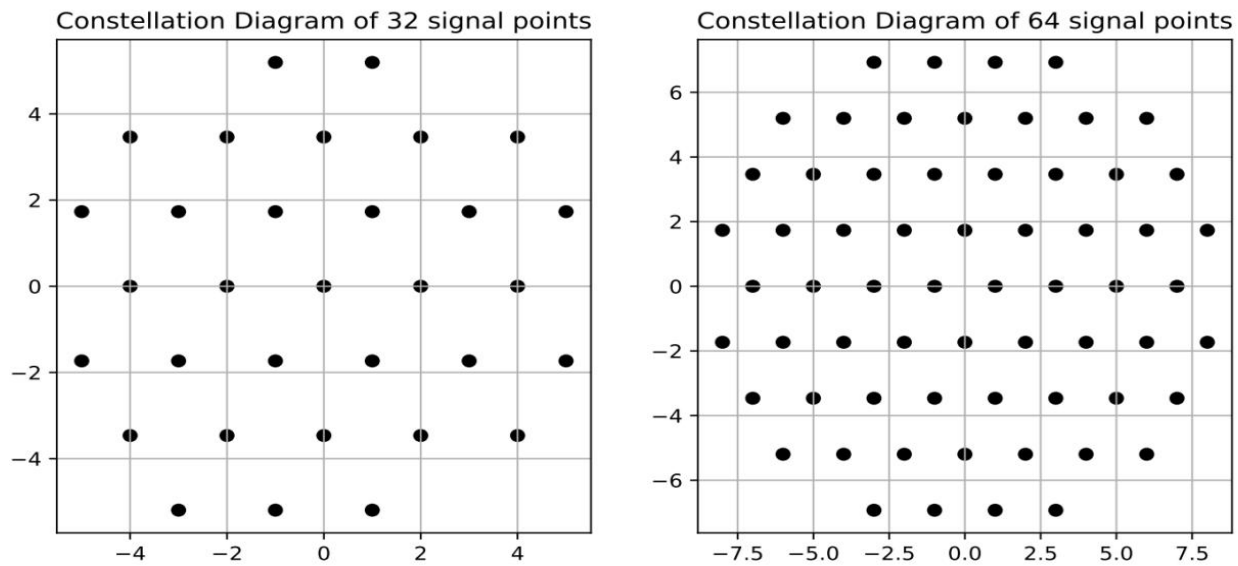

Fig. 4. Constellation Diagram at 32 and 64 points.

At this point, you may find that the contour of the whole Constellation Diagram is somewhat circular. Indeed, the more points, the closer the contour of the PLC Constellation Diagram is to the circle. For example, when the number of points is 1024 , the contour of the constellation in Fig. 5 is very close to the circle, which is the inevitable result of making the signal point as close as possible to the origin of the coordinate.

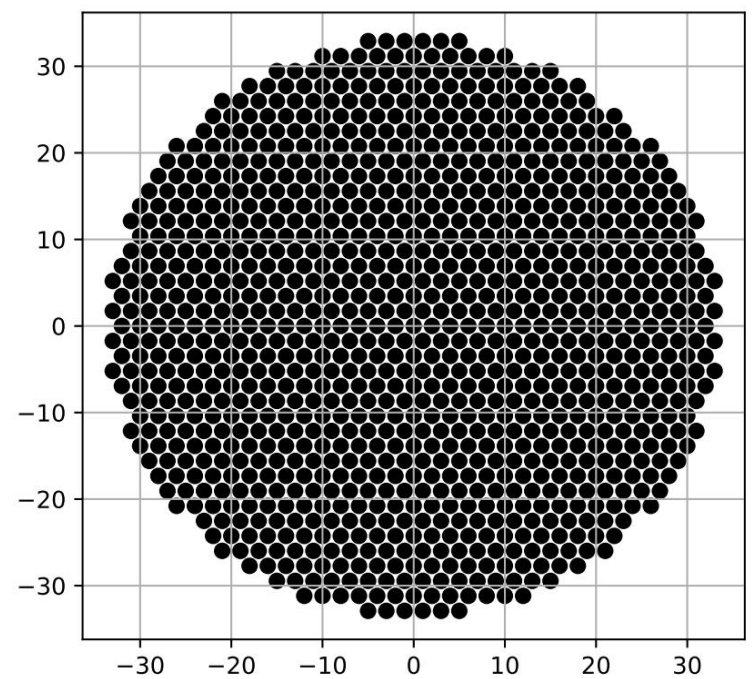

Fig. 5.Constellation Diagram of 1024 signal points.

When the number of points $\mathrm{N}$ is large, the relative error of any two of the four schemes will be very small (the number will be smaller as the number of points increases), and there are: 


$$
\lim _{n \rightarrow \infty} \frac{E_{N-P L C}}{E_{N-Q A M}}=1
$$

In addition, we sometimes need to follow the above general method of Constellation Diagram planning based on the actual situation and not necessarily follow the above. For example,

we can plan the Constellation Diagram in a square area like a square QAM. In this way, we ensure that each row has the same number of signal points, just as the QAM Constellation Diagram is staggered between rows and rows, and then refer to the equilateral triangular grid for design. In this way, while improving the Space Utilization Rate, it can also take the QAM's Gray code characteristics into account. Here we show the Constellation Diagram of Square QAM and Square PLC over 64 points in Fig 6. If you are careful enough, you will find that the red points and blue points in the square PLC Constellation Diagram can be obtained by stretching the vertical axes of the two QAM Constellation Diagrams. Therefore, when analyzing or implementing the system, you can refer to QAM and you can get it with a slight change.

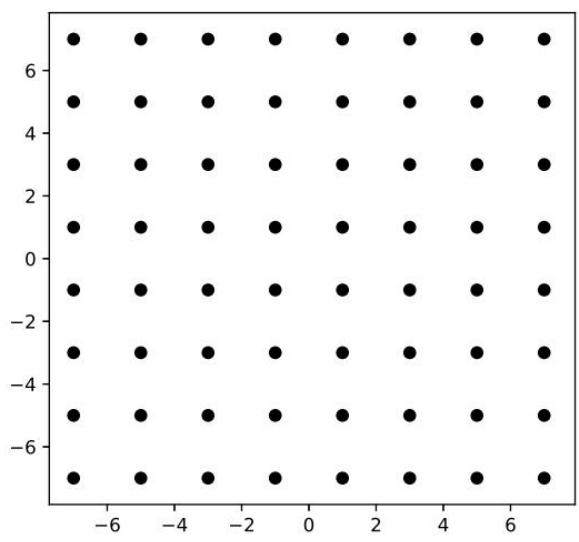

(a)Square QAM Design

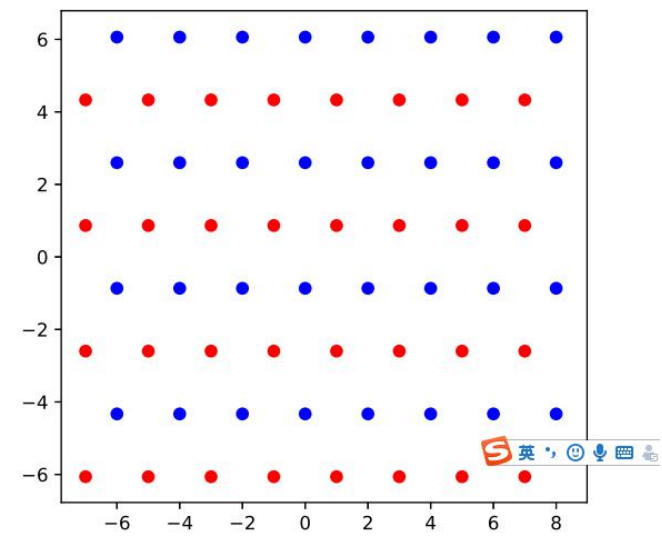

(b) Square PLC Design

Fig. 6. Constellation Diagram of Square QAM and Square PLC over 64 points

Besides, the Performance Analysis of Constellation Diagrams below are based on generally arranged model. The square constellations or other variants are described only, and no further analysis is provided below.

\section{Performance Analysis}

For the following energy analysis and SER analysis, we set the distance between adjacent signal points is 2 . 


\subsection{Energy Analysis}

We still keep the distance between the adjacent signal points equal to 2. Let's first observe the average energy changes of N-QAM and N-PLC from 2 to 2048, as shown in Fig. 7.

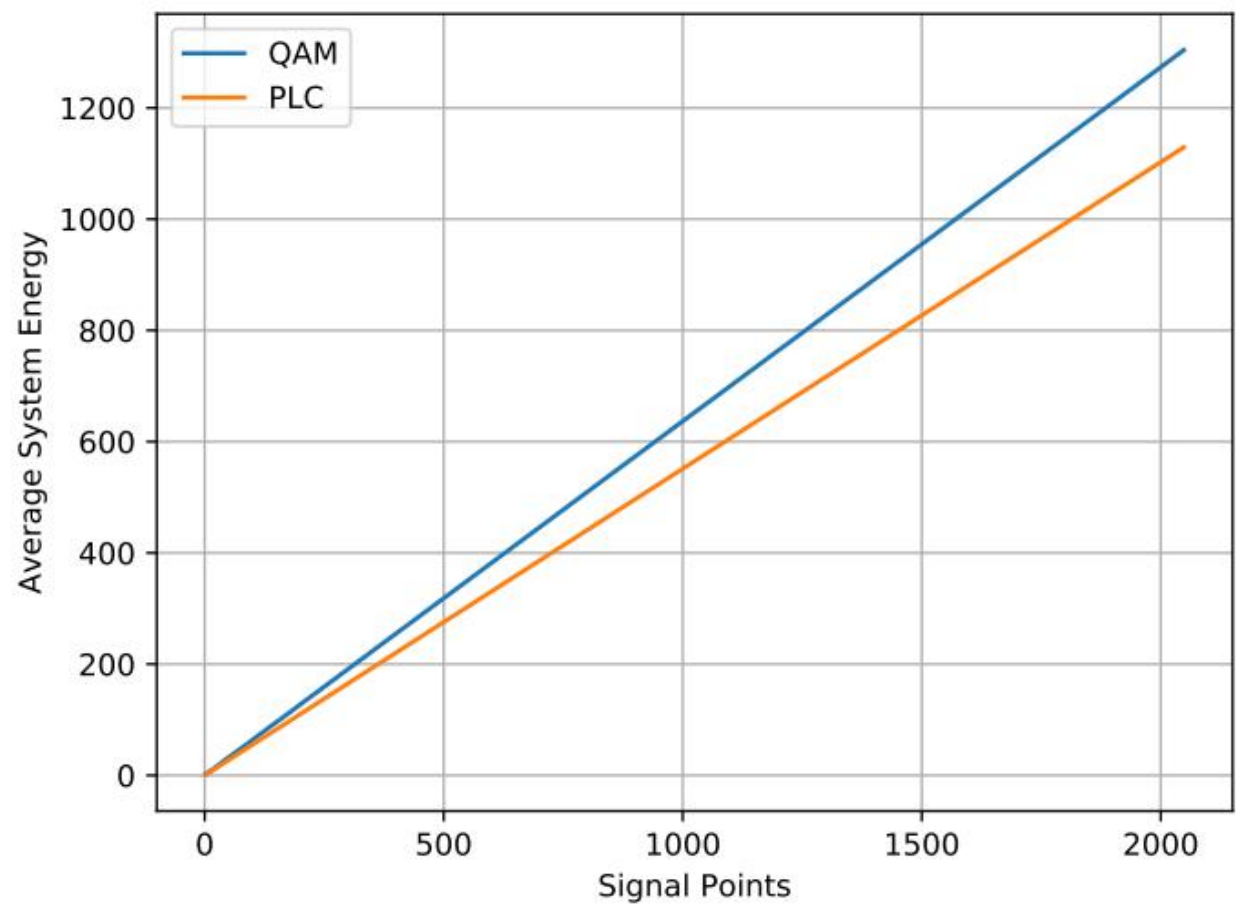

Fig. 7. The Variation of Average System Energy with Signal Points

We find that the average energy of the two schemes increases approximately linearly as the number of points increases. And the average energy of PLC is lower than that of NQAM, which preliminarily reflects the effect of hexagonal arrangement method to reduce the Average System Energy. In addition, the energy estimation function at $\mathrm{N}$ point is given here (where the distance between signal points is set as $2 \mathrm{~A}$ )

$$
\begin{aligned}
& E_{Q A M}(N) \approx 0.6367 N A^{2} \\
& E_{P L C}(N) \approx 0.5513 N A^{2}
\end{aligned}
$$

We can see from (5) and (6) that:

$$
\frac{E_{P L C}}{E_{Q A M}}=\frac{\sqrt{3}}{2} \approx 0.8660
$$

More specifically, the variation of the ASE ratio of N-PLC and N-QAM systems in the range of 2 to 2048 is given in Fig. 8 . 


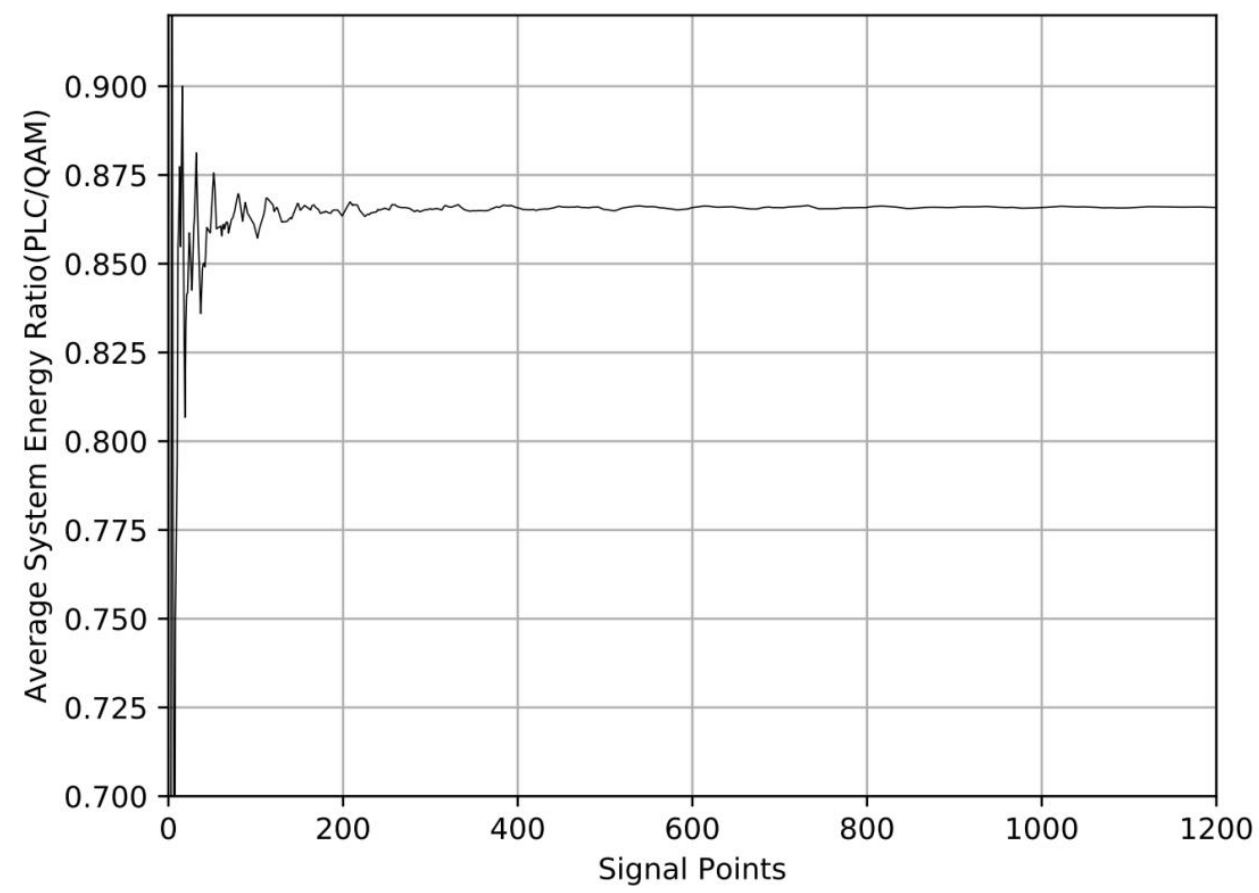

Fig. 8. The Variation of ASE ratiowith Signal Points

When there are few signal points, the graph fluctuates sharply because the characteristic of arrangements is not obvious.But the arrangement model become more obvious with the increase of the number of signal points, the ratio of the ASE of the two schemes tends to be stable after 130 points, which is about 0.8660 , that is, the ratio of their SUR.

Table 2. Performance comparison when the number of signal points is 2 integer power.

\begin{tabular}{|c|c|c|c|}
\hline \multirow{2}{*}{ Bit Number } & \multicolumn{2}{|c|}{ ASE } & \multirow{2}{*}{ ASE Ratio(N-PLC/N-QAM) } \\
\hline & N-PLC & N-QAM & \\
\hline 1 & 1.000 & 2.0000 & 0.5000 \\
\hline 2 & 2.000 & 2.0000 & 1.0000 \\
\hline 3 & 4.5000 & 6.0000 & 0.7500 \\
\hline 4 & 9.0000 & 10.0000 & 0.9000 \\
\hline 5 & 17.6250 & 20.0000 & 0.8813 \\
\hline 6 & 35.2500 & 41.0000 & 0.8598 \\
\hline 7 & 70.5625 & 81.7500 & 0.8631 \\
\hline
\end{tabular}




\begin{tabular}{llll}
\hline 8 & 141.0469 & 162.7500 & 0.8666 \\
9 & 282.1406 & 326.1875 & 0.8650 \\
10 & 564.5508 & 651.7813 & 0.8662 \\
11 & 1129.0918 & 1303.9375 & 0.8659 \\
12 & 2258.2090 & 2607.5938 & 0.8660 \\
13 & 4516.4722 & 5215.1406 & 0.8660 \\
\hline
\end{tabular}

In practical applications, we often take the positive integer power of 2 as the number of points to design the communication system. In this paper, the ASE change table in the case of $\mathrm{N}$ power of 2 in given in Table.2.

Though Energy comparison, we find that the design of Constellation Diagram of communication system by using hexagonal arrangement method can ensure that the distance between signal points remains unchanged, so that the system has lower ASE. In other words, if the ASE is the same, the hexagonal arrangement can make the allowance between signal points more specific, thus improving the anti-noise performance.

\subsection{SER analysis under AWGN}

Let the independent random variables $\mathrm{X}$ and $\mathrm{Y}$ have the same variance $\sigma^{2}$. If $\mathrm{X}$ and $\mathrm{Y}$ obey the two-dimensional normal distribution, their probability density function is :

$$
f(x, y)=\frac{1}{2 \pi \sigma^{2}} e^{-\frac{x^{2}+y^{2}}{2 \sigma^{2}}}
$$

And more, if the Energy of AWGN is $E=\sigma_{x}^{2}+\sigma_{y}^{2}=2 \sigma^{2}$, the probability density function can also be described as :

$$
\varphi(x, y)=\frac{1}{\pi E} e^{-\frac{x^{2}+y^{2}}{E}}
$$

Set the distance between adjacent signal points is $2 \mathrm{~A}$. If each received signal point is decided to the nearest standard signal point, we can think that the decision range of each signal point is the circumscribed regular polygon of the circle with the radius of $A$ and the signal point as the center.For example, the Decision Area of Square Arrangement and Hexagonal Arrangement, surrounded by red curves, is shown in Fig.9. 

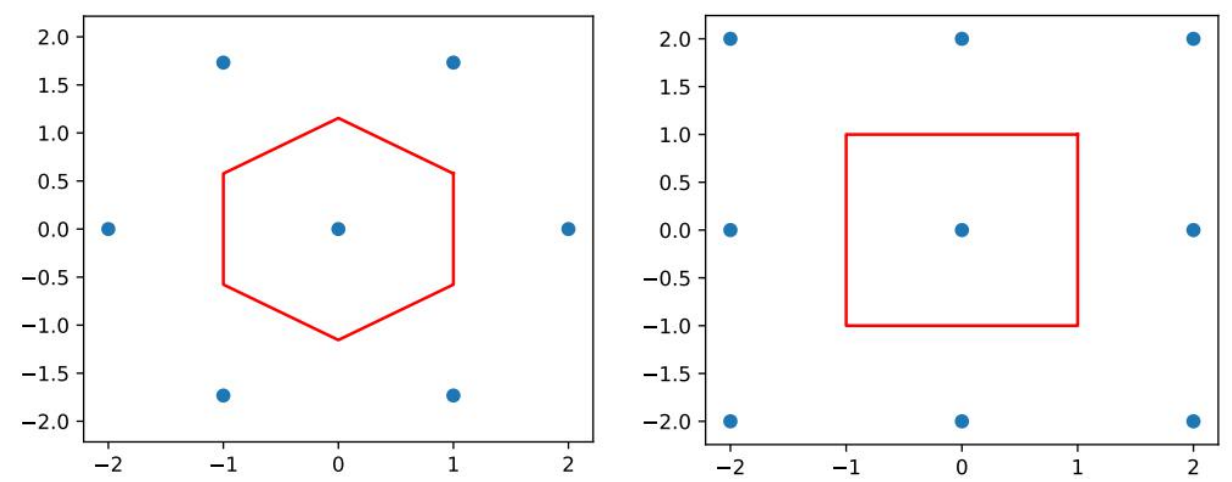

Fig. 9. Decision Area of Square Arrangement and Hexagonal Arrangement

Let the decision area is $\mathrm{C}$, then the SER is the probability of $(\mathrm{X}, \mathrm{Y})$ appears out of $\mathrm{C}$ and it can be calculated by

$$
S E R=1-\iint_{C} \frac{1}{\pi E} e^{-\frac{x^{2}+y^{2}}{E}} d x d y
$$

For the following discussion, let the distance between adjacent signal points of Square QAM is 2. Slightly enlarge the distance between adjacent signal points in PLC, which has lower ASE, to make the Energy of them the same. According to equation (5) and (6).

$$
0.5513 \cdot A_{P L C}^{2}=0.6367 \cdot 1^{2} \Rightarrow A_{P L C} \approx 1.0747
$$

In above case compare their SER according to equation (10), the SER variation is shown in Fig. 12.

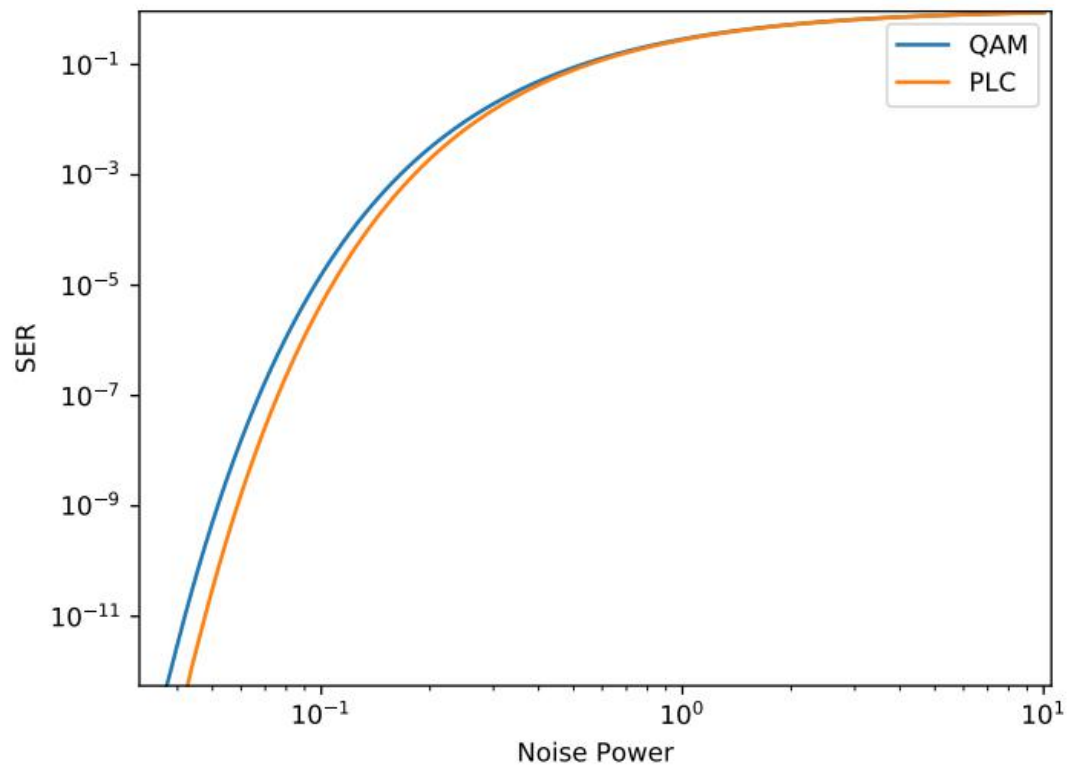

Fig. 10. SER Variation of PLC and QAM under AWGN 
We can see that as the noise power becomes small, the SER becomes lower, and the SER of PLC is lower than SER of QAM. Besides, the optimization effect of PLC is more obvious in low noise power case.

\section{Summary and Outlook}

In this paper, the Constellation Diagram of communication system is designed to improve the SUR. It improves the power utilization efficiency and reduces the ASE or SER. And with the increase of the number of points, the energy optimization effect tends to be stable with the SER ratio approach to 0.8660 . Besides, the SER of PLC is lower when ASE are the same. The automatic Constellation Diagram design algorithm in this paper provides a solution for readers to sort out ideas and solve the problem of large amount of calculation when the number of points is high, and fills the blank of the existing literature in this respect. Besides, if we slightly enlarge the distance of signal points in PLC to make its energy equals to QAM, the PLC model has lower SER.

The conceptual approach and method in this paper provides a new idea for the research and design of Constellation Diagrams. At the same time, they are of great significance to the research and design of high-dimensional Constellation Diagrams. Although the technology in this paper fully embodies the advantages of reducing the average energy of the system, there are only four schemes for choosing the center point, so that we can get a good but not the best center point. Next, we intend to study the precise selection of the optimal center point algorithm and the design of high-dimensional Constellation Diagram, so as to make the theory more perfect.

\section{References}

[1] Jian-Xin W , Hui S. "Digital modulation recognition based on constellation diagram," Journal of China Institute of Communications, vol. 25, no. 6, pp. 166-173, June 2004.

[2] Yiling W, Hongbin L, Yuping Z. "A Novel Constellation Design for $2^{2 \mathrm{n}+1}$-QAM". Journal of Electronics and Information, vol. 32, no. 6, pp. 1510-1514, Jun. 2010.

[3] Yadan Zhang. Design and implementation of OFDM system based on ni-usrp [D]. 2016(in Chinese)

[4] B. Chen, M. Jiang. "A Method of Constructing Three-dimensional Constellation Map of Communication System," China, CN201410593718.6[P].2015-01-28.(in Chinese)

[5] Hao W, Jianqiu Z, Hanbin S. "A lattice based approach to the construction of multi dimensional signal constructions," Journal of Electronics, vol. 42, no. 9 , pp. 1672-1679, Sep. 2014.

[6] Fang H , Pan C , Yang Z . "Reducing the OFDM peak-to-average power ratio with improved constellation extension," Journal of Tsinghua University (Science and Technology), vol. 45, no. 10, pp.1373-1376, Oct. 2005.

[7] S. Tharranetharan, M. Saranraj, S. Sathyaram and V. R. Herath, "A performance comparison of nonlinear phase noise tolerant constellation diagrams," 6th International Conference on Industrial and Information Systems, Kandy, pp. 439-442, Aug.2011.

[8] F. Huang, X. Liu, Z. Zhou, J. Luo and J. Wang, "Quadrature Index Modulation With ThreeDimension Constellation," in IEEE Access, vol. 7, pp. 182335-182347, Dec.2019. 